\title{
Structure and stellar populations in early-type galaxies
}

\author{
Alexandre Vazdekis ${ }^{1}$, Ignacio Trujillo ${ }^{2}$ and Yoshihiko Yamada ${ }^{3}$ \\ ${ }^{1}$ Instituto de Astrofísica de Canarias, E-38200 La Laguna, Tenerife, Spain email: \\ vazdekis@ll.iac.es \\ ${ }^{2}$ Max-Planck-Institut für Astronomie, Königstuhl 17, D-69117 Heidelberg, Germany \\ ${ }^{3}$ National Astronomical Observatory of Japan, 2-21-1 Osawa, Mitaka, Tokyo 181-8588, Japan
}

\begin{abstract}
We explore possible correlations between the light profile shapes of early-type galaxies, as parameterized by the Sérsic index $n$, and relevant stellar population parameters, which were obtained from spectra of extremely high signal-to-noise and stellar population models that provide an age indicator that is virtually free of the effects of metallicity. We do not find any robust correlation between $n$ and mean age or metallicity. However, we find evidence for a strong positive correlation between $n$ and $[\mathrm{Mg} / \mathrm{Fe}]$ abundance ratio. We speculate that earlytype galaxies settle up their structure on short time-scales in agreement with those imposed by their $[\mathrm{Mg} / \mathrm{Fe}]$ ratios, suggesting that the global structure was already at place at high $z$.
\end{abstract}

\section{Introduction}

Early-type (E/SO) galaxies follow tight relations such as the Fundamental Plane, the Color-Magnitude Relation or the $\mathrm{Mg}_{2}-\sigma$ relation (Bender et al. 1992). However little is known about the relation between their morphological shapes and stellar populations (Conselice 2003), most likely because these two fields rely on methods that have grown up separately. Aiming at exploring possible correlations between galaxy light profiles and their stellar populations, we combine results obtained from analyzing high-quality spectroscopic data and stellar light profile fitting as modelled by the Sérsic index $n$ (see for details Vazdekis, Trujillo \& Yamada 2004).

\section{Galaxy measurements}

We use the mean luminosity weighted ages, metallicities $([\mathrm{M} / \mathrm{H}])$ and abundance ratios $([\mathrm{Mg} / \mathrm{Fe}])$ derived by Vazdekis et al (2001) and Yamada et al. (2004a,b in preparation) for a sample of Virgo and field early-type galaxies. Flux-calibrated spectra of extremely high signal-to-noise $(\mathrm{S} / \mathrm{N}>100)$ from galaxy central regions $\left(r_{e} / 10\right)$ were used. Relevant line-strengths, were measured and compared to those predicted by the model of Vazdekis (1999). Ages were determined on the basis of the $\mathrm{H} \gamma_{\sigma}$ age indicator of Vazdekis \& Arimoto (1999), which is free from metallicity effects.

The Sérsic shape parameters for most of Virgo galaxies were taken from Caon et al. (1993), or by fitting a Sérsic model to published (Peletier et al. 1990) or derived light profiles (HST archive images). We also use the concentration index of Trujillo et al. (2001) $C_{r e}(1 / 3)$, which evaluates the fraction of light enclosed within $r_{e} / 3$ divided by the light within $r_{e}$, and is monotonically related to $n$. 


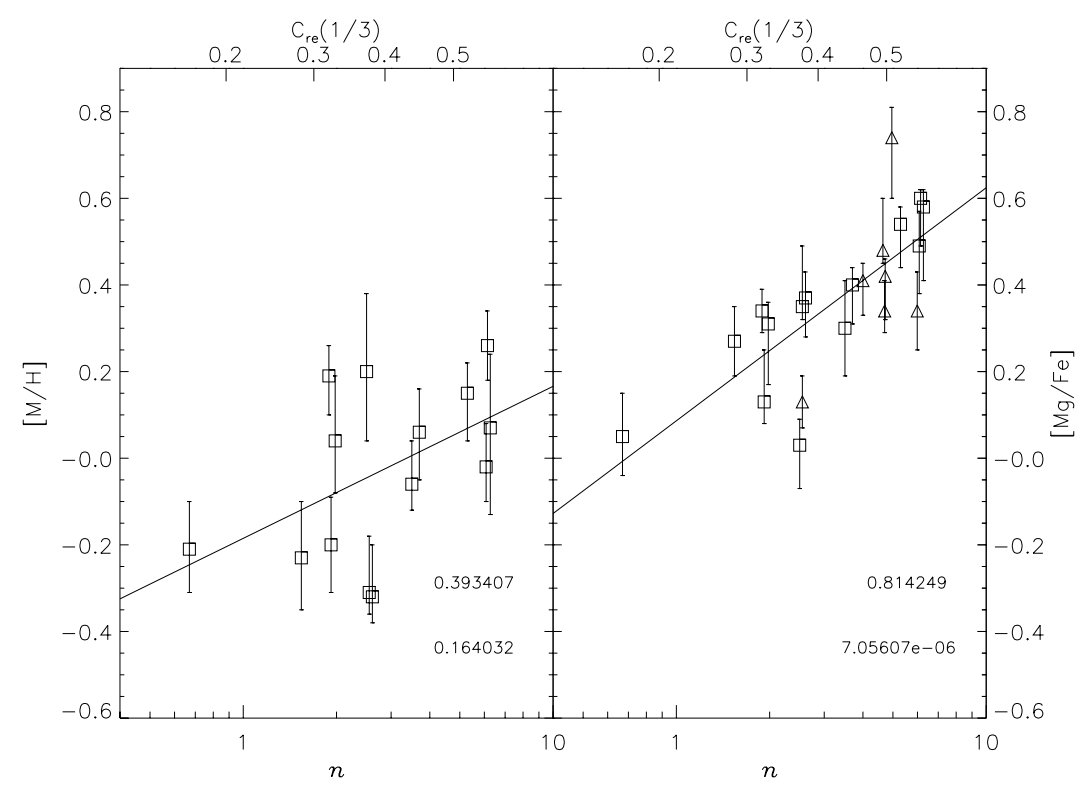

Figure 1. Plot of the shape parameters, $n$ and $C_{r e}(1 / 3)$, versus $[\mathrm{M} / \mathrm{H}]$, and $[\mathrm{Mg} / \mathrm{Fe}]$. Squares mean Virgo galaxies and triangles mean field galaxies. Spearman rank-order correlation coefficient and its significance value are written in each panel.

\section{Results}

No correlation can be found between galaxy ages and $n$ as most of our galaxies are rather old (Vazdekis et al. 2001). Fig. 1 (left panel) shows a weak correlation (as shown by the Spearman test $r_{s}$ ) between the total metallicity and the shape parameters. However, Fig. 1 shows for the first time a strong positive correlation between $[\mathrm{Mg} / \mathrm{Fe}]$ abundance ratio and the shape parameter indices. This result is expected since $[\mathrm{Mg} / \mathrm{Fe}]$ correlates with $\sigma$ (Trager et al. 2000), and $\sigma$ correlates with $C_{r e}(1 / 3)$ (Graham et al. 2001). Nonetheless, it is surprising to see that the $[\mathrm{Mg} / \mathrm{Fe}]$ provides the strongest correlation among other stellar population parameters, reaching a Spearman coefficient 0.8. Finally, similar correlation values are obtained for the already known $C_{r e}(1 / 3)-\sigma$ and $[\mathrm{Mg} / \mathrm{Fe}]-\sigma$ relations on the basis of the same galaxies, probing the robustness of the new correlation.

\section{Discussion}

$\mathrm{Mg}$ is ejected to the ISM by SN II on short time-scales $\left(\leqslant 10^{6}-10^{7} \mathrm{yr}\right)$. If star formation stops before SN Ia release Fe $(\sim 1 \mathrm{Gyr})$, the $[\mathrm{Mg} / \mathrm{Fe}]$ should be larger than 0 . The central $\sigma-[\mathrm{Mg} / \mathrm{Fe}]$ correlation suggests that the innermost regions of more massive galaxies stopped their star formation on short time-scales. Next, the fact that $n$ correlates with $[\mathrm{Mg} / \mathrm{Fe}]$ suggests that early-type galaxies settled up their global structure (and not only the innermost regions) on time-scales according to their $[\mathrm{Mg} / \mathrm{Fe}]$ (a scenario favored if no $[\mathrm{Mg} / \mathrm{Fe}]$ radial gradients were present, Mehlert et al. 2003). The fact that we do not find a correlation between $n$ and galaxy mean ages but $[\mathrm{Mg} / \mathrm{Fe}]$ suggests that the formation time-scale is the main process linked to galaxy global structure, rather than burst peppering as a function of time. Since our galaxies are old and the time scales of most massive galaxies very short, the new correlation suggests that the global structure was already at place at high $z$, without experiencing a significant time evolution. 


\section{Acknowledgements}

We would like to thank N. Caon and H.-W. Rix for useful suggestions and J. Blakeslee for providing us with photometric data for NGC 4489 and NGC 7454. This research is based on observations made with the European Southern Observatory telescopes obtained from the ESO/ST-ECF Science Archive Facility.

\section{References}

Bender, R., Burstein, D., Faber, S.M. 1992, ApJ, 399, 462

Caon, N., Capaccioli, M. \& D'Onofrio, M., 1993, MNRAS, 265, 1013

Conselice, C. 2003, ApJSS, 147, 1

Graham, A., Trujillo, I., Caon, N. 2001, AJ, 122, 1707

Mehlert, D., Thomas, D., Saglia, R., Bender, R., Wegner, G. 2003, A\&A, 407, 423

Peletier, R., Davies, R., Illingworth, G., Davis, L., Cawson, M., 1990, AJ, 100, 1091

Trager, S., Faber, S., Worthey, G., González, J. 2000, AJ, 120, 165

Trujillo, I., Graham, A., Caon, N. 2001, MNRAS, 326, 869

Vazdekis, A. 1999, ApJ, 513, 224

Vazdekis, A., Trujillo, I., Yamada, Y. 2004, ApJ, 601, L33

Vazdekis, A., Kuntschner, H., Davies, R., Arimoto, N., Nakamura, O., Peletier, R. 2001, ApJ, 551, L127

Vazdekis, A., Arimoto, N. 1999, ApJ, 525, 144 